\title{
Marktwirtschaft und Soziale Gerechtigkeit
}

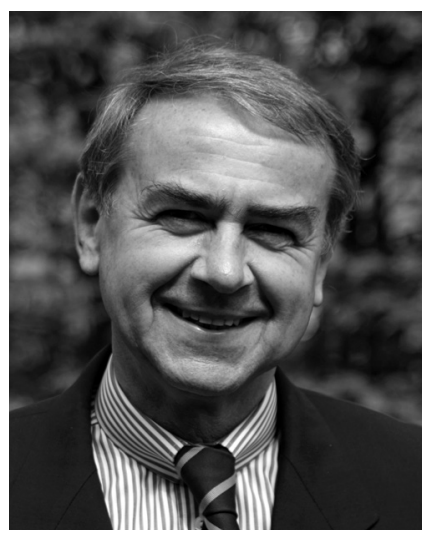

VON PETER EICHHORN

Prof. Dr. Dr. h.c. mult. Peter Eichhorn war von 1981 bis zu seiner Emeritierung im Jahre 2007 Professor für Betriebswirtschaftslehre, insbesondere Public \& Nonprofit Management an der Universität Mannheim. Heute ist er u. a. Vorsitzender des wissenschaftlichen Beirats des OPINIO Forschungsinstitut Sonja McClain \& Joachim Merk GbR, einem Beratungsinstitut für das Gesundheitswesen.

www.opinio-forschungsinstitut.de

\author{
Gerade im sozialen Bereich gibt es eine \\ Gratwanderung zwischen Einzelfallgerechtigkeit \\ und ausufernder Bürokratie. Eine Rückbesinnung \\ auf die Grundlagen von Sozialer Marktwirtschaft \\ und das Sozialstaatsgebot des Grundgesetzes kann \\ bei der Bestimmung des richtigen Maßes helfen.
}

Mit Bürokratismus, einer übersteigerten Form von Bürokratie, verbindet man umständliche und langwierige Verfahren, denen man ausgeliefert ist und deren Verfahrenszwänge keine Auswege eröffnen.

Die Macht der Bürokraten äußert sich darin, dass sie den Arbeits- und Zeitaufwand bei sich und ihrem Gegenüber beeinflussen können. Obwohl an Regelwerke formal und inhaltlich gebunden, nutzen Bürokraten die erheblichen Spielräume bei der Verfahrensabwicklung. Rückfragen, Auskünfte, Begründungen, Nachweise usw. bieten ausreichend Gelegenheit, behördliche Herrschaft auszuüben. Der britische Historiker Cyril Northcote Parkinson (1909-1993) fand für das unhaltbare Wuchern der Bürokratie die Ursache heraus und kleidete sie in das Parkinsonsche Gesetz: Arbeit dehnt sich in dem Maße aus, wie Zeit $\mathrm{zu}$ ihrer Erledigung verfügbar ist.

Bürokratismus kennt regelmäßig keine Knappheit an Zeit. Deshalb können sich Bürokraten ganz und gar absichern, anders formuliert: anfechtbare Risiken ausschließen. Bürokraten gehen auf Nummer sicher.

Folgerichtig resultieren daraus Rechte für das Amt und Pflichten für den Antragsteller, Steuerzahler usw. Im Bürokratismus ist es zulässig und ein probates Mittel, selbst einen einfach gelagerten Vorgang penibel zu hinterfragen, Informationen aus Archiven, Grundbüchern, Dokumenten, Plänen akribisch zusammenzutragen, Bedarfe zu erforschen, die einschlägige Rechtsprechung zu Rate zu ziehen, die aktuelle Rechtsentwicklung abzuwarten, Kollegen zu befragen, Vorgesetzte um Prüfung zu bitten, Dienstwege nicht abzukürzen, sondern ausführlich zu gehen.

Falls möglich, wird Verwaltungsarbeit auf Bürger und Unternehmen abgewälzt; gern beauftragt man auch externe Dritte und beschäftigt damit Berufsstände wie Buchprüfer, Steuerberater, Wirtschaftsprüfer, Rechtsanwälte, Unternehmensberater, Architekten, Bauingenieure, Umweltexperten und andere Sachverständige.

Ein drastisches Beispiel, wie Bürokratismus aus sich selbst heraus wächst, liefert die Rentenversicherung in Deutschland. Diese Mammutbehörde beschäftigt derzeit rund 63.000 Mitarbeiter. Sie zählt damit zu den größten öffentlichen Dienstherren. Das komplizierte Rentenrecht, das dem Individualprinzip huldigt und keine Pauschalen für Beitragsaufkommen und Rentenzahlungen kennt (wie das in anderen Ländern üblich ist), hat aber nicht nur Rentenberater für die Rentenleistungen hervorgebracht. Neben den Leistungsbereichen für das Publikum (aktuelle und künftige Rentner) mit internen und externen Auskunfts- und Beratungsstellen wurden Widerspruchsstellen und Widerspruchsausschüsse gegen Rentenbescheide etabliert, die dem sozialgerichtlichen Verfahren vorgelagert sind. Hinzu kommen Rechtsmittelstellen zur Vorbereitung von Klagen an den Sozialgerichten über die Landessozialgerichte bis hin zum Bundessozialgericht. Damit soll nicht die Sozialgerichtsbarkeit als solche moniert werden, wohl aber die 
ständigen Änderungen unterworfene, komplizierte und auslegungsbedürftige Rentengesetzgebung, die exzessive Rechtsprechung hervorruft, welche zwingend zu bürokratischer Zusatzarbeit führt.

In Verbindung mit dem sich ändernden Rehabilitationsrecht findet Bürokratismus außer in der Deutschen Rentenversicherung Bund, der Deutschen Rentenversicherung Knappschaft-Bahn-See und 14 Rentenversicherungen der Länder auch in den mit ihnen kooperierenden Arbeitsverwaltungen, Krankenkassen und Berufsgenossenschaften und deren Unfallkliniken statt. Rentenversicherungseigene Reha-Krankenhäuser vorwiegend für Suchtkranke, Psychiatrie und Orthopädie sollen dafür sorgen, dass die Arbeitsfähigkeit von Patienten erhalten wird, um vorzeitige Rentenfälle zu verhindern. Vorzeitige Rentenbegehren bearbeiten die Sozialmedizinischen Dienste.

Die Betriebsprüfdienste bei den 16 Trägern der Rentenversicherung mit 3.600 Mitarbeitern im Außen- und Innendienst wiederum sind notgedrungen erforderlich, um die sozialabgabepflichtigen Betriebe zu beraten und zu prüfen, ob und inwieweit die Lohnunterlagen, Abrechnungen und Arbeitsverträge mit dem übereinstimmen, was die Arbeitgeber gemeldet haben. Der Kontrolle unterliegen die Beiträge zur Renten-, Kranken-, Pflege- und Arbeitslosenversicherung sowie beispielsweise die Umlagen für Lohnfortzahlungen und Mutterschaftsgeld. Zu den Kontrollaufgaben der Betriebsprüfer gehören auch seit 2007 die Abgaben zur Künstlersozialversicherung, seit 2010 die Insolvenzgeldumlagen und seit 2011 die Beiträge zur Unfallversicherung. Als im Jahr 2011 das Bundesarbeitsgericht die »Tarifgemeinschaft Christlicher Gewerkschaften für Zeitarbeit und Personalserviceagenturen « für tarifunfähig erklärte, werden jetzt Zeitarbeitsfirmen vom Betriebsprüfdienst befragt, ob die von ihnen vertretenen Leiharbeiter nach dem Tarif der Entleihfirmen entlohnt werden.

Damit diese teils zentralen, teils regionalen Rentenversicherungsapparate flächendeckend funktionieren, bedarf es außer den Leistungsabteilungen mit ihren Dezernaten großer Verwaltungsabteilungen. Sie befassen sich mit Grundsatzfragen, Koordination und Öffentlichkeitsarbeit, mit Controlling, Haushalt, Datenverarbeitung und Zentralarchiven, Bau, Betrieb und Unterhaltung der Bü- rogebäude, Konferenzräume, Kantinen, Tiefgaragen, Parkhäuser, Höfe und Gartenanlagen, mit Ausschreibung, Auftragsvergabe und Rechnungsprüfung.

Dem riesigen Personalkörper dient ein aufwendiges Personalmanagement mit ungezählten Stellen für Aus- und Fortbildung der Mitarbeiter (die aufgrund laufender Rechts- und Bürokratieänderungen sich laufend qualifizieren müssen) sowie für die Bearbeitung von Beihilfen, Reise- und Umzugskosten; Teilzeitregelungen, Elternzeitanforderungen und Wiedereingliederungsprogramme gilt es zu beachten und umzusetzen bis hin zu Kinderferien- und Notfallbetreuung. Gleichstellungs- und Integrationsbeauftragte und Personalräte binden weitere Kräfte, wobei deren Tätigkeiten nicht an sich zu kritisieren sind, sondern nur bezüglich ihres Umfangs, denn die Erfahrung lehrt: Je größer eine Institution, desto mehr Personen und höhere Kosten für Personalsteuerung und Mitbestimmung. Überproportional wachsen mit der Betriebsgröße auch die zeitaufwendigen und damit kostspieligen Besprechungen zwischen den Beschäftigten in den Zentralen und bei und mit den Regionalträgern. über 46 Millionen Euro - wählen nach Listen mit Namen von Organisationen; die Personen bleiben im Hintergrund. Die gewählten Vertreter verabschieden zwar die Haushaltspläne und wählen als Aufsichtsgremien die Vorstände, über die Rentenformel aber entscheidet die Politik. Für wenig Kompetenz eine teure Wahlfarce!

\section{Soziale Arbeit}

Die Tätigkeit von Sozialarbeitern der kommunalen Jugend- und Sozialämter widmet sich Personen in gesellschaftlichen Notlagen, welche sie allein nicht meistern können. Die Hilfebedürftigkeit ist weit gespannt. Sie reicht von familiären Konflikten über die Misshandlung und Verwahrlosung von Kindern, Schulschwänzen, Alkoholmissbrauch, Drogensucht, Spielleidenschaft, Disziplinlosigkeit, berufliche Verweigerung, soziale Desintegration, Arbeitslosigkeit, unzureichende Wohnverhältnisse, Verschuldung, Straffälligkeit und Ausgrenzung bis hin zu materieller Not.

Durch Sozialarbeit sollen die Betroffenen wieder in die Gesellschaft integriert werden. Deshalb ist Sozialarbeit auch Krisenintervention, Dienst am Menschen und

\section{"Parkinson hat recht: Arbeit dehnt sich in genau dem Maß aus, wie Zeit für ihre Erledigung zur Verfügung steht"}

Niemand kennt die echten Verwaltungskosten samt Abschreibungen, Pensions- und sonstige Rückstellungen für die Massenverwaltung der einzelnen Rentenfälle. Zu ihnen zählen auch die einschlägigen Gesetzgebungs-, Gerichts- und Rechnungshofkosten, letztere für die Ordnungsmäßigkeits-, Rechtmäßigkeits- und Wirtschaftlichkeitsprüfungen. (Was die Prüfung der Wirtschaftlichkeit anbelangt, werden aber nur Kosten im Rahmen vorgegebener Leistungen untersucht, nicht hingegen, was es weniger kosten würde, wenn man statt individueller Leistungen Pauschalen einführen würde.)

Den Gipfel des bürokratischen Systems der Rentenversicherung bilden alle sechs Jahre wiederkehrende Sozialwahlen für die Gremien zur Selbstverwaltung. Lediglich ein Drittel von 46 Millionen Wahlberechtigten - bei geschätzten Ausgaben von
Lobbying für Arme, Behinderte, Diskriminierte, Gefährdete, Notleidende und sozial Isolierte. Sozialarbeiter bemühen sich darum, die Ursachen menschlicher Notlagen zu beseitigen, indem sie beraten, betreuen, fördern, individuelle Perspektiven aufzeigen und Verhalten einüben.

Als Instrumente der Sozialarbeit haben sich bewährt: Unterstützung bei der Selbsterkennung und Selbsthilfe, Beratung und Begleitung des Hilfebedürftigen durch den Alltag, Kommunikation mit ähnlich gelagerten "Fällen « und soziale Gruppenarbeit einschließlich Unterbringung in Familien und Heimen mit Therapien, Unterstützung der Gruppenselbstorganisation, Netzwerkbildung in der Verwandtschaft, Nachbarschaft und in der Form institutioneller Kontakte mit Bildungsstätten, Arbeitsagenturen, Entzugseinrichtungen, Obdachlosenhei- 


\section{Was man unter Bürokratie versteht}

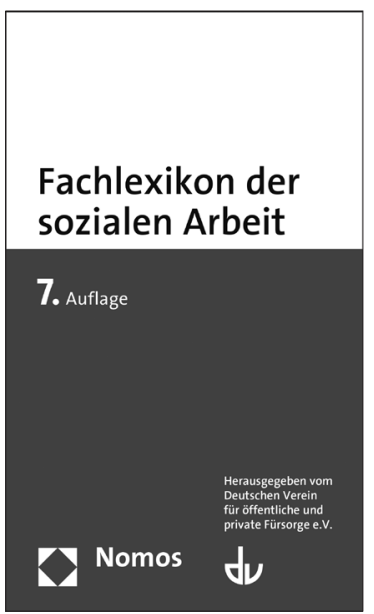

1. Unter Bürokratie wird eine spezifische Ausprägung des modernen Beamtentums und des Verwaltungshandelns in öffentlich-rechtlichen wie privaten Organisationen verstanden. Insbesondere durch das Werk des Soziologen Max Weber liegt eine ausgearbeitete Bürokratie-Theorie vor. Charakteristisch für die Bürokratie ist die Herausbildung von spezialisierten Aufgaben aufgrund fachlicher Ausbildung. Zentrale Elemente sind ihre Regelgebundenheit, die Amtshierarchie mit festgelegten Zuständigkeiten, das Laufbahnprinzip und v. a. die unparteiische und objektive Erledigung von Aufgaben ohne Ansehen der Person. Die bürokratische Organisation zeichnet sich idealtypisch aus durch geschultes Personal, Einheitlichkeit, Unterordnung sowie Vermeidung von Reibungsverlusten und Herstellung effizienter Geschäftsabläufe. Mit diesen Charakteristika setzte sie sich gegenüber allen anderen Organisationsformen durch. Da Bürokratie somit Regeln, Zwecke, Mittel und sachliche Unpersönlichkeit zur Voraussetzung hat, ist sie "rationalen « Charakters und deshalb eng verkoppelt mit dem für die Entwicklung kapitalistischer Wirtschaftsformen besonders förderlichen Prinzip des "Rationalismus".

2. In der Alltagssprache wird Bürokratie häufig gleichgesetzt mit langwierigen, umständlichen und kleinlichen Verfahrensweisen mit oft lebensfremden Ergebnissen, eine Sichtweise, die sich sowohl auf öffentliche Verwaltungen als auch auf private Unternehmen bezieht.

3. Derzeit befinden wir uns in einem institutionellen Wandel, der mit den Begriffen Verwaltungsmodernisierung, New Public Management (NPM), neues Steuerungsmodell oder neue Formen des Governance gekennzeichnet wird. Vom theoretischen Standpunkt aus wird die Verwaltung nicht mehr ausschließlich als "rationale", sondern als Organisation mit »begrenzter Rationalität (bounded rationality) angesehen, die ihren eigenen Gesetzmäßigkeiten unterworfen ist. Im Unterschied zur Bürokratie-Theorie Webers liegt gleichwohl kein konsistenter theoretischer Entwurf vor. Stattdessen wird vielfach auf "Best-Practice-Modelle "Bezug genommen, indem Erfahrungen erfolgreicher Organisationen im Hinblick auf eigene Zielvorstellungen adaptiert werden. Wichtiges Resultat dieser Neuori- entierung ist, dass die Verwaltung nicht mehr nur durch präzise Rechtsnormen oder zweckgebundene Mittelzuweisungen gesteuert wird, sondern durch Ziel- und Leistungsvereinbarungen (ZLV), durch Outputsteuerung anstelle von Inputsteuerung und durch Globalhaushalte, bei denen die Verwaltung selbst entscheidet, wie sie ihre Mittel am zweckmäßigsten zur Erreichung der vereinbarten Ziele einsetzt. Über Zielund Leistugsvereinbarungen werden inhaltliche Vorgaben für das Handeln der Verwaltung operationalisiert. Ein wesentliches Merkmal neuer bürokratischer Organisationen liegt deshalb in der Veränderung von einer strikten Verhaltens- hin zur Ergebniskontrolle (Controlling), die anhand der Zielerreichung, nach Qualitätskriterien oder Verfahren der Leistungsbemessung durchgeführt wird. Eine grundlegende Änderung betrifft darüber hinaus das Selbstverständnis der betroffenen Beamten/ Angestellten: Waren sie im bürokratischen Modell nur nach hierarchischen Anweisungen tätig, übernehmen sie im Falle des New Public Managements die vollständige Verantwortung sowohl für ihre Entscheidungen wie die Effizienz ihres Handelns.

\section{Rolf von Lüde}

Quelle: Deutscher Verein für öffentliche und private Fürsorge e. V. (Hg.): Fachlexikon der sozialen Arbeit. 7. Auflage. Nomos Verlagsgesellschaft, Baden-Baden 2011. 1.139 Seiten. 44,- Euro. ISBN 978-3-8329-5153-5. men, Kirchen, Caritas, Diakonie und weiteren Wohlfahrtsträgern.

Sozialarbeit ist Wertschöpfung pur, weil humaner Nutzen gestiftet wird. Es findet sogar monetäre Wertschöpfung (»Sozialprofit«) statt, denn resozialisierte Personen reduzieren Sozialausgaben und zahlen zumindest als Berufstätige Steuerund Sozialabgaben.

\section{Sozialmanagement}

Dieser inzwischen eingebürgerte Begriff umfasst das teils verbindliche, teils freiwillige Erstellen und Anbieten von Sozialleistungen. Ihr hauptsächlicher Zweck sind der soziale Ausgleich, insbesondere die Beseitigung von sozialen, medizinischen und ökologischen Missständen, und die Verhütung von sozialen Notlagen. Obwohl der Begriff zunächst vorwiegend in
Sozialunternehmen seinen Eingang fand, gehört er inzwischen zum alltäglichen Sprachgebrauch von Sozialverwaltungen auf allen Ebenen.

Ausgehend vom Sozialstaatsprinzip, einer in Artikel 20 und 28 Grundgesetz normierten generalklauselartigen Staatszielbestimmung, kümmern sich die für soziale Angelegenheiten zuständigen Arbeits-, Bildungs-, Familien-, Gesundheits-, Jugend- und Sozialministerien des Bundes und der Länder um Sozialpolitik im weiteren Sinn der Einkommensumverteilung durch soziale Sicherungssysteme.

Sozialmanagement besteht dann in der Evaluation, Konzeption und Implementation der Sozialpolitik. Vom Sozialmanagement erwartet man zum Beispiel Risikoanalysen von Alter und Krankheit, Untersuchungen der Ausbildungssituation von Jugendlichen, der Langzeitar- beitslosigkeit, der Lebensumstände von Alleinerziehenden, jungen und älteren Migranten - und von den Ergebnissen Ansätze für die Finanzierung der Geldleistungen (z. B. Elterngeld und andere Transferleistungen), Sachleistungen (z. B. Medikamente, Unterkunft) und Dienstleistungen (z. B. Beratung, Betreuung) und deren Leistungskombination (z. B. Gewährung von Sozialhilfe, Ausbau der Kinderkrippen).

Je nach Geschäftsverteilung und Organisation werden solche Managementaufgaben von obersten oder oberen Behörden erledigt. Das Sozialmanagement beispielsweise eines Landesamtes für Soziales als Oberbehörde erstreckt sich darüber hinaus auf zahlreiche und vielfältige Aufgabengebiete: die Betriebserlaubnis für Kindertagesstätten, die Jugendhilfe, die Integration von Menschen 
mit Behinderungen, die Aufsicht über Altenheime und Krankenkassen usw.

Auf der kommunalen Ebene betreiben die Sozialämter (häufig auch Jugendund Sozialämter) Sozialmanagement vor allem durch die Sozialarbeiter. So schwärmen "Streetworker " in die "Problembezirke« der Städte aus, Fachkräfte in Allgemeinen Sozialen Diensten helfen unter anderem den gesundheitlich Angeschlagenen, verwahrlosten Kindern, süchtigen Singles, sozial Isolierten, finanziell Schwachen, Jugendlichen und Familien in schwierigen Lebenslagen.

Das Management der Sozialarbeiter wandelt sich von der Beratung zur Intervention. Da Eingriffe auch Rechte von Dritten betreffen, ist besonders sorgfältige Schreibtischarbeit gefordert, gepaart mit vertrauensbildenden Maßnahmen.

Die interne Organisation der Sozialämter richtet sich gewöhnlich nach den im Bundessozialhilfegesetz (SGB XII) vorgesehenen Leistungsarten, nämlich nach den verschiedenen Hilfen zum Lebensunterhalt und in besonderen Lebenslagen. In Verbindung mit weiteren Hilfen, Ansprüchen und Teilhaberechten, auch von Seiten anderer Ämter, ist es wegen der Vielzahl von beteiligten Stellen und Spezialisten für Leistungsberechtigte kompliziert, Sozialleistungen zu beantragen und tatsächlich zu empfangen.

Aus Nachfragersicht empfiehlt sich eine Reorganisation, die den Publikumsverkehr auf Kundengruppen ausrichtet. Diese können nach öfters auftretenden Merkmalen von Personen gebildet werden. Minderjährige Aussiedler ohne ausreichende deutsche Sprachkenntnisse und ohne Ausbildungsplatz fasst man zum Beispiel zusammen und lässt sie von einem Sachbearbeiter betreuen. Die Bedienung aus einer Hand erweist sich als kundenfreundlich, setzt aber bei den Bediensteten voraus, dass sie die einschlägigen Leistungsbündel kennen und $\mathrm{zu}$ handhaben verstehen.

\section{Sozialökonomie}

Nomen est omen. Der Name deutet auf die Ökonomisierung hin: Das Sozialwesen wird wirtschaftlich durchdrungen wie es ähnlich in anderen Lebensbereichen geschieht. Man spricht auch von Bildungs-, Gesundheits-, Hochschul-, Militär-, Prozess- und Verwaltungsökonomie und löst jeweils den allgemeinen und unbestimmten Wesensbegriff $a b$.
Doch was verbirgt sich hinter dieser spezifischen Begrifflichkeit? Es sind wohl sechs Aspekte, die man als begriffsimmanent bezeichnen kann (und die häufig für mit Ökonomie oder Wirtschaft zusammengesetzte Phänomene analog gelten):

- Die Sozialökonomie (synonym: Sozialwirtschaft) nimmt eine intermediäre Stellung ein zwischen Gesellschaft, Staat, Markt und Natur. Sie reicht in alle Aggregate hinein und wird von ihnen beeinflusst. Um es kurz auszudrücken: Die Gesellschaft respektive Gemeinschaft bedarf ihrer, der Staat setzt die Regeln, der Markt bildet den Aktionsraum und die Natur fordert ihr Recht.

- Die Sozialökonomie ist ein beachtlicher Wirtschaftsfaktor. Auf sie entfallen in den unterschiedlichen gemeinnützigen und gewerblichen Sozialunternehmen schätzungsweise zehn Prozent aller Arbeitsplätze in Deutschland und ebenso in den EUMitgliedsländern. Durch ihre Eigentümer- und Trägervielfalt, die diversen
Rechtsformen, die dezentrale Struktur, die kleine bis mittlere Betriebsgröße und den meist örtlichen Bezug findet sie in der Wirtschaftspresse allerdings wenig Resonanz. Lokalzeitungen berichten eher über Notstände und Missmanagement, aber auch über Engagement und »Tag der offenen Tür«, statt auf die Bedeutung für stabile Beschäftigung, Einkommen und Wertschöpfung hinzuweisen.

- Von der Sozialökonomie werden soziale Dienstleistungen erbracht. Diese Produkte zeichnen sich durch unmittelbare Tätigkeiten zwischen Menschen (face to face communication) aus und zwar derart, dass Hilfebedarf im weiten Sinn gedeckt wird. Die Erstellung und die Abgabe der Sozialen Arbeit erfolgen meist »uno actu«, erfordern Präsenz oft auch nachts und am Wochenende und richten sich auf Schwache in unserer Gesellschaft, nämlich auf Kinder und Jugendliche, Kranke, Behinderte, Alte, Arme Alleinerziehende, Arbeitslose, Zuwanderer usw.

\section{Von 28 auf über 2.000 Wörter: das Finanzierungssys- tem der Behinderten- und Altenhilfe}

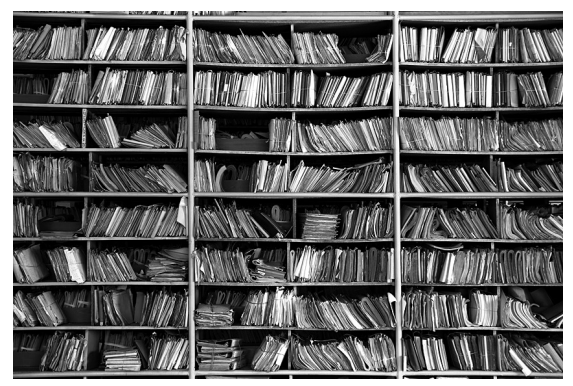

Das Auseinanderklaffen von Absicht und Realität in Bezug auf Entbürokratisierung ist nicht neu. Das Bundeskabinett hatte am 19. März 1970 einen Beschluss gefasst, wonach »die Kodifizierung eines Sozialgesetzbuchs auf der Grundlage der sozialen Wertentscheidungen des Grundgesetzes mit dem Ziel angestrebt wird, das Sozialrecht für die Bevölkerung überschaubarer zu machen und seine Durchführung für die Verwaltung zu vereinfachen «. Zwei Beispiele zur Umsetzung dieser gehegten Absicht:

- Werkstättenmitwirkungsverordnung: Die Mitwirkungsrechte der Beschäftigten in Werkstätten für behindert Menschen war in $\S 14$ der Werkstättenverordnung geregelt, also in einem einzigen Paragrafen. Ergänzt wurde 2001 dieser $\$ 14$ von der 41 Paragrafen umfassenden
Werkstättenmitwirkungsverordnung die zu beachtende Rechtsmasse ist auf das 180-fache angewachsen.

- Finanzierungssystem der Behinderten- und Altenhilfe: Von 1961 bis 1984 basierte dies auf einem einzigen Satz mit 28 Wörtern. Von 1984 bis 1994 galt ein konkretisierter Absatz mit dann 150 Wörtern, um dem Sozialhilfeträger zu ermöglichen, vom Träger der Einrichtung Wirtschaftlichkeit und Sparsamkeit verlangen zu können. Zwischen 1994 und 1999 galt eine Übergangsfassung, die sechs Absätze mit 450 Wörtern umfasste. Anfang 1999 bis Ende 2004 trat eine neue Fassung in Kraft, die fünf Paragrafen enthielt mit 16 Absätzen und über 1470 Wörtern. Die Rechtsmasse wurde damit verfünfzigfacht, und zwar im Rahmen einer sich beschleunigenden Gesetzgebung.

Der zu regelnde Gegenstand hingegen blieb in seinem Wesensgehalt unverändert der gleiche. Konsequent fortgesetzt wurde der eingeschlagene Weg in dem heute gültigen SGB XII. Hier sind es jetzt sieben Paragrafen mit 19 Absätzen und über 2.000 Wörtern

Quelle: Zeitschrift SOZIALwirtschaft 3/2006 
- In der Sozialökonomie wird zum großen Teil hybrid finanziert. Bund, Länder und Gemeinden, freie Wohlfahrtsträger und private Stiftungen stellen laufende oder einmalige Zuschüsse (u. a. Beihilfen, Beiträge, Fördermittel, Subventionen) bereit. Im Übrigen werden Entgelte erhoben, deren Höhe davon abhängt, ob administrative (»soziale «) Preise vorgegeben sind oder sich die Preiskalkulation an der Wettbewerbslage orientiert. Der Regelfall dürfte die Mischfinanzierung sein. Die Sozialunternehmen, Sozialunternehmer und »Social Entrepreneurs « zählen dann gewöhnlich zum Non-ProfitSektor. Für ihn ist typisch, dass auf der Gewinnentstehungsseite zumindest auf Kostendeckung (durch Zuschüsse plus Entgelte) Wert gelegt wird und etwaige Überschüsse der Selbstfinanzierung (für Wachstum) dienen. Soweit gemeinnützige und steuerlich begünstigte Zwecke verfolgt werden, gilt ohnehin ein Gewinnausschüttungsverbot. In Verbindung mit einer starken Marktstellung, beispielsweise veranlasst durch eine lokale oder regionale Monopolsituation oder eine hohe Reputation, kann dies im äußersten Fall zu einer Aufwandmaximierung führen. Großzügige Gehälter und Honorare, prestigeträchtige Bauwerke und Parkanlagen, preziöse Ausstattungen und Fahrzeuge weisen in diese Richtung.

- Ein weiteres Kennzeichen sozialökonomischer Betätigung ist die Gesinnungsgemeinschaft. Sinngebundenes Wirtschaften äußert sich im Idealfall in gemeinwohlorientierter Hingabe, solidarischem Verhalten, ethischem Vorbild und in der Betonung von Gleichheit und Gleichstellung. Die Motivation mag in religiösen Anliegen, kirchlichen oder kulturellen Bedingungen wurzeln oder weltanschaulich oder rein pragmatisch begründet sein. Gegen diese privatwirtschaftliche Alternative polemisieren indessen gern jene Sozialunternehmer, die ihre Dienste und Einrichtungen schlicht gewinnstrebend betreiben.

- Gemeinwohlorientierung bedeutet in der Sozialökonomie zugleich auch ökologische Nachhaltigkeit. Man will die Natur bewusst so nutzen, dass sie erneuerungsfähig bleibt, natürliche Ressourcen sparsam verbraucht werden und Schadstoffe möglichst nur im Rahmen der Absorptionsfähigkeit durch die Umwelt entstehen.

\section{Sozialstaatsgebot}

Ein in Artikel 20 des Grundgesetzes verankerter elementarer Verfassungsgrundsatz verpflichtet in erster Linie den Gesetzgeber dazu, gesellschaftliche Gegensätze und Konflikte auszugleichen, insbesondere wirtschaftlich Schwache zu schützen, in Not geratenen Bürgern zu helfen und ihnen ein menschenwürdiges Dasein und eine angemessene Beteiligung am allgemeinen Wohlstand zu gewähren. Das Sozialstaatsgebot bildet den anderen Pol der Sozialen Marktwirtschaft mit ihren grundgesetzlich und größtenteils auch europarechtlich gesicherten Freiheiten wie der Konsumfreiheit, Gewerbefreiheit, Freiheit der Berufs- und Arbeitsplatzwahl, Produktions- und Handelsfreiheit sowie der Freiheit der Eigentumsnutzung.

Die allgemein gehaltene Formulierung des Sozialstaatsgebots oder Sozialstaatsprinzips trifft keine genaueren inhaltlichen Bestimmungen, so dass sich im Regelfall keine konkreten Einzelansprüche ableiten lassen. Rechtlich fassbare Begriffe und justitiable Forderungen enthalten die Gesetze zur Vermeidung von Ungleichheiten in den sozialen Verhältnissen. Aber auch beim Gesetzesvollzug stößt man auf Ermessensentscheidungen bei der Abwägung und Auslegung.

Dem vorrangigen Ziel, soziale Notlagen und Beeinträchtigungen durch Krankheit, Invalidität, Arbeitslosigkeit und Alter zu bewältigen, widmen sich die Sozialversicherungen (d. h. die gesetzliche Kranken-, Pflege-, Unfall-, Arbeitslosen- und Rentenversicherung). Das Sozialstaatsgebot schlägt sich darüber hinaus in zahlreichen Gesetzen und Verordnungen nieder für die Fürsorge für Hilfsbedürftige, für die Kriegsopferversorgung und Kriegslastenverteilung, zum Arbeitsschutz (insbesondere Sicherheit des Arbeitsplatzes und der Arbeitszeit), zur Daseinsvorsorge (kollektive Existenzsicherung durch Infrastrukturmaßnahmen), zur Bildungs-, Gesundheitsund Wohnungsbauförderung bis hin zum Schutz der Wirtschaftsdemokratie (insbesondere durch Mitbestimmungsgesetze und Gesetze gegen Wettbewerbsbeschränkungen). Eine Ausprägung des Sozialstaatsgebots ist auch die Sozialbindung des Eigentums (Art. 14 Abs. 2 Grundgesetz: »Eigentum verpflichtet. Sein Gebrauch soll zugleich dem Wohle der Allgemeinheit dienen.«).
Die gesetzlichen Sozialversicherungen, behördlichen Sozialhilfen und die genannten weiteren Sozialleistungen, Fördermaßnahmen und Regelungen zur Verhütung des Missbrauchs wirtschaftlicher Machtstellung gelten als wesentlicher Bestandteil der Sozialen Marktwirtschaft in Deutschland. Das Kennzeichen dieser Wirtschaftsordnung ist der Ausgleich zwischen der persönlichen und wirtschaftlichen Entfaltung im Wettbewerb und der finanziellen Absicherung der Menschen durch Korrekturen der Marktergebnisse (mittels Sozialbeiträgen, Steuern, Subventionen, Unterstützungen usw.).

Eine Ausweitung des Sozialstaatsgebots ist in Deutschland möglich und wird zur Eindämmung der globalen Finanz- und Wirtschaftskrise praktiziert, ohne allerdings beim Namen genannt zu werden. Angesprochen sind die derzeitigen (Teil-) Verstaatlichungen von (systemrelevanten) Banken und anderen (Schlüssel-) Unternehmen. Im Grunde wendet man den »Vorratsartikel « 15 des Grundgesetzes an, wonach »Produktionsmittel « (zu denen beispielsweise auch Banken gehören) »zum Zwecke der Vergesellschaftung durch ein Gesetz, das Art und Ausmaß der Entschädigung regelt, in Gemeineigentum oder in andere Formen der Gemeinwirtschaft überführt werden können.«

\section{Resümee}

Es fehlt nicht an Versuchen, das Sozialstaatsgebot über seinen bisherigen $\mathrm{Ka}$ talog hinaus auf weitere Gebiete auszudehnen und als Grundrechte in die Verfassung aufzunehmen. Beispielhaft genannt seien das Recht auf Arbeit und das Recht auf Wohnen.

Manche befürchten, andere befürworten, dass ein umfassendes Sozialstaatsgebot zu einer sozialistischen Markt- oder gar Planwirtschaft führt. Dies würde geschehen, wenn das Privateigentum nicht mehr respektiert und die dezentral gelenkte Wirtschaftsordnung aufgegeben würde.

Der vorstehende Artikel ist ein redaktionell bearbeiteter Wiederabdruck aus der Zeitschrift »Deutsche VerwaltungsPraxis« 7/2012. 